\title{
Study of the Mechanical Properties and Vibration Isolation Performance of a Molecular Spring Isolator
}

\author{
Muchun Yu, Xue Gao, and Qian Chen \\ State Key Laboratory of Mechanics and Control of Mechanical Structures, Nanjing University of Aeronautics and Astronautics, \\ Nanjing 210016, China
}

Correspondence should be addressed to Qian Chen; q.chen@nuaa.edu.cn

Received 6 March 2016; Revised 27 April 2016; Accepted 8 May 2016

Academic Editor: Nuno M. Maia

Copyright (C) 2016 Muchun Yu et al. This is an open access article distributed under the Creative Commons Attribution License, which permits unrestricted use, distribution, and reproduction in any medium, provided the original work is properly cited.

Molecular Spring Isolator (MSI) is a novel passive vibration isolation technique, providing High-Static-Low-Dynamic (HSLD) stiffness based on the use of molecular spring material. The molecular spring material is a solid-liquid mixture consisting of water and hydrophobic nanoporous materials. Under a certain level of external pressure, water molecules can intrude into the hydrophobic pores of nanoporous materials, developing an additional solid-liquid interface. Such interfaces are able to store, release, and transform mechanical energy, providing properties like mechanical spring. Having been only recently developed, the basic mechanic properties of a MSI have not been studied in depth. This paper focuses on the stiffness influence factors, the dynamic frequency response, and the vibration isolation performance of a MSI; these properties help engineers to design MSIs for different engineering applications. First, the working mechanism of a MSI is introduced from a three-dimensional general view of the water infiltration massive hydrophobic nanoporous pores. Next, a wide range of influence factors on the stiffness properties of MSI are studied. In addition, the frequency response functions (FRFs) of the MSI vibration isolation system are studied utilizing the matching method based on equivalent piecewise linear (EPL) system. Finally, the vibration isolation properties of MSI are evaluated by force transmissibility.

\section{Introduction}

Due to the advantages of simple structure, high reliability, relatively lighter weight, and low cost compared to active vibration isolation, passive vibration isolation technologies are being intensively studied and developed in various industrial areas. A variety of isolators, for example, coil spring, rubber isolator, metal rubber isolator, steel cable isolator, and air spring isolator, are applied to various industrial sectors. However, in the area of heavy equipment vibration isolation, there are still some difficult issues to address, such as large static deflection, unstable performance, material aging, and gas leakage (from air springs). As a result, researchers in the vibration isolation area are still seeking new technologies and innovations [1-3].

Conventionally, nanoporous materials, such as zeolites and silica gel, have a wide range of applications in adsorption, separation, ion-exchange, heterogeneous catalysis, and other desired functions. Recently, a new field of application involving the energetics of nanoporous materials has been developed [4]. It has been found that, depending on the external pressure, water molecules can intrude into the nanopores of hydrophobic nanoporous materials. As shown in Figure 1, during the compression process, an external pressure $P$ works against the Laplace capillary pressure $P_{c}$, which tends to push out water molecules

$$
P_{\mathrm{c}}=-\frac{2 \sigma \cos \theta}{r}
$$

where $\sigma$ is the surface tension of water, $\theta$ is the contact angle of the water and solid interface, and $r$ is the radius of pore [5]. As long as $P$ overcomes $P_{c}$, water molecules intrude into the nanopores.

When the hydraulic pressure decreases, different types of hydrophobic nanoporous materials exhibit different behaviours. For materials such as hydrophobic silica gel, water molecules remain in the nanoenvironment. Thus, there is a hysteresis of force for which the external energy is almost 


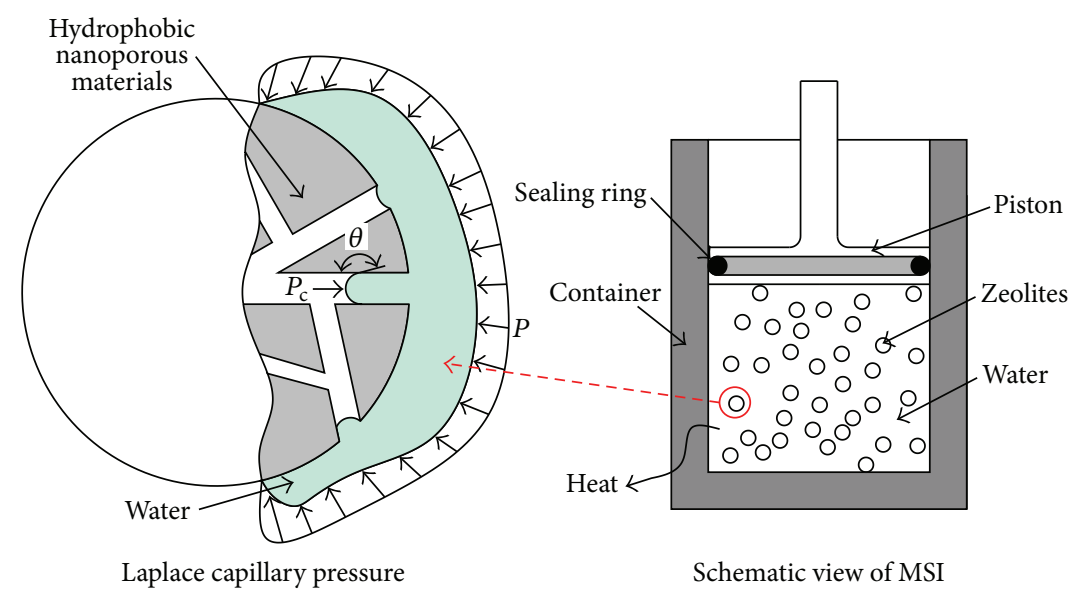

(a)

(b)

FIGURE 1: Water intrudes into hydrophobic nanopores under external pressure; (b) is the structure of a Molecular Spring Isolator (MSI), and (a) is the schematic view of Laplace capillary pressure.

completely absorbed during the loading-unloading process [5-11]. Therefore, the mixture of water and hydrophobic silica gel can be used as a damper; as a result, it is named colloidal damper (CD) [5-7], nanocolloidal damper (NCD) [8], or nanoporous energy absorption system (NEAS) [9-11]. In contrast, for materials such as hydrophobic zeolites, water molecules extrude from the cavities automatically as soon as the hydraulic pressure drops; that is, the extrusion is the inverse process of intrusion. Thus, a reciprocal transformation of mechanical energy into interfacial energy occurs with very low energy loss. As the mixture of water and hydrophobic zeolites exhibits the property of a spring, it is termed as molecular spring [12-17]. The causes of the differences between the behaviours of $\mathrm{CD}$ (or NCD and NEAS) and the molecular spring are still not clear, but it is thought to be related to the diameter of the hydrophobic pores $[18,19]$. Moreover, silica gel obtains the hydrophobicity by modifying the surface with a linear chain of $n$-alkylchlorosilanes [5], whereas the zeolites of molecular spring are inherently hydrophobic because of the all-silica framework. This distinction may be another reason of the difference between $\mathrm{CD}$ and molecular spring.

This work integrates molecular spring into a High-StaticLow-Dynamic (HSLD) stiffness isolator. For a linear isolator system, the stiffness of its isolator spring should be as low as possible to increase the frequency bandwidth within which the vibration isolation comes into effect. However, low stiffness isolator normally induces unacceptably large static deflection. Thus, there is a trade-off between isolation frequency range and static deflection of traditional linear isolators [20]. Recent studies show that a molecular spring exhibits HSLD stiffness intrinsically $[12,13,21]$ and is able to support a heavy static load with very low dynamic stiffness. As a result, the molecular spring has a potential to develop into a novel technology for use in vehicle suspensions, engine vibration isolation, and so on.

It has been affirmed that several types of zeolites, such as Silicalite-1, SSZ-24, ZSM-22, ZSM-12, DD3R, and Si-CHA, can be used as the nanoporous material for a molecular spring [12-15]. This paper studied a Molecular Spring Isolator (MSI) of a cylinder-piston structure that is filled with Silicalite-1 zeolite as the medium. In our former work, the mechanical model of this MSI was established, and a few influence factors on the stiffness were introduced. In this work, first, the working mechanism of the molecular spring is briefly presented, based on a three-dimensional general view of the water infiltration of a massive number of hydrophobic nanoporous pores. Subsequently, to provide design principles for MSI under different engineering applications, a wider range of influence factors on stiffness is investigated. Furthermore, to study the dynamic properties of MSI, the frequency response functions (FRFs) of a single degree of freedom system supported by a MSI are analyzed using the matching method based on an equivalent piecewise linear (EPL) system. The vibration isolation properties of MSI are also evaluated by force transmissibility.

\section{Mechanical Properties}

2.1. Working Mechanism. As defined by IUPAC (International Union of Pure and Applied Chemistry), the pores whose diameter is less than $2 \mathrm{~nm}$ is termed a micropore [22]. Water molecules will be forced into hydrophobic micropores, depending on the external pressure, and expelled from micropores when hydraulic pressure decreases. That mechanism is the working principle of molecular spring.

Figure 2 shows a comparison of the quasistatic experimental stiffness of the MSI with that of pure water. The MSI is found to exhibit piecewise-nonlinear stiffness. The stiffness of the MSI is comprised of three stages-static stiffness stage, dynamic stiffness stage, and stopping stiffness stage. In the static stiffness stage, the external pressure is insufficient to overcome the Laplace capillary pressure; thus, the working mechanism of this stage is compression of water. Therefore, 


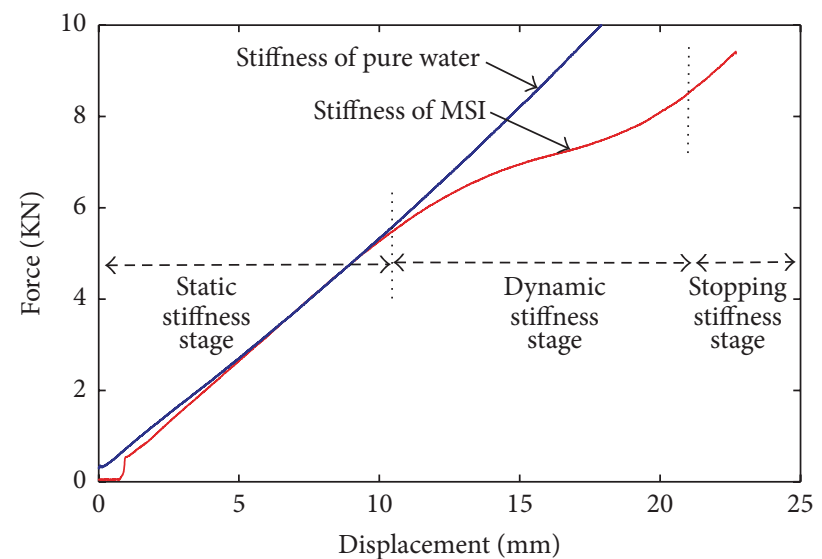

FIGURE 2: Experimental stiffness of the MSI and the compression of pure water.

the MSI exhibits very high static stiffness, and the forcedisplacement curve of this stage coincides with that of the compression of pure water.

Water starts to intrude into hydrophobic pores during the dynamic stiffness stage. Considering that water infiltration leads to a large volume change, if the size and the contact angles of the pores are constant, then the force-displacement curve of the dynamic stiffness stage will exhibit a horizontal plateau. However, in practice, the pores of zeolites vary in both size and hydrophobicity, especially for the commercial Silicalite-1 zeolites used in this work, depending on the characteristics of the zeolites. The aperture of zeolites is supposed to be a uniform distribution, and the contact angle is assumed to be a Gaussian distribution within certain ranges [21]. As a result, under external pressure, water molecules first fill the pores that have the largest apertures and the smallest contact angles and then fill the smaller pores with larger contact angles. Figure 3 shows a three-dimensional general view of the water infiltration massive hydrophobic nanoporous pores. The Laplace capillary pressure surface corresponds to the Laplace capillary pressure of each pore with different radius and contact angle. The Laplace capillary pressure surface is intersected by constant pressure planes. When the external pressure rises to the level of the constant pressure plane, the pores whose Laplace capillary pressures are under the constant pressure plane are filled with water. The pores above the constant pressure plane still avoid water. Finally, if the pressure is large enough, then all of the pores become saturated with water. Therefore, the forcedisplacement curve exhibits a low slope in dynamic stiffness stage.

After all of the pores are saturated, that is, the stopping stiffness stage, the working mechanism returns to be the compression of water. Thus, the stiffness becomes rigid again, as shown in Figure 2.

2.2. Influence Factors on the Stiffness. The quantity of zeolites and temperature are proved to have an influence on the stiffness of a MSI [21]: (1) the dynamic stiffness softens when the number of zeolite particles increases and the static

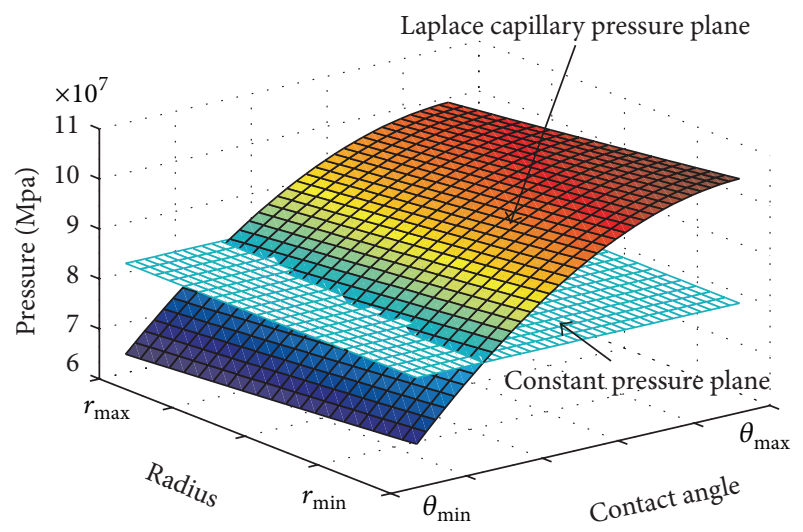

FIGURE 3: Intersection of the Laplace capillary pressure surface and the constant pressure planes $\left(\theta_{\min }\left(\theta_{\max }\right)\right.$ and $r_{\min }\left(r_{\max }\right)$ are the minimum (maximum) value of the contact angle and radius, resp.).

stiffness remains unchanged; (2) the working pressure of the dynamic stiffness stage decreases with the temperature rising. To design the stiffness properties of a MSI for different engineering applications, more influence factors should be studied.

In the macroview, it is obvious that the quantity of zeolites and the cross-sectional area of the piston will have influence on the stiffness. In the microview, the surface tension $\sigma$ and the contact angle $\theta$ will affect the process of water infiltration according to (1); hence, the correlation factors influence the stiffness. Accordingly, the hydrophobicity of the material, the temperature, and the presence of ions or surface active agents in water are also supposed to be influence factors. The influences of the quantity of zeolites and temperature have been introduced in [21]; as supplementary work, in this section, the other influence factors are investigated by experiments or theoretical analysis. In addition, considering that the properties of $\mathrm{CD}$ change during the endurance test [6], an endurance test of the MSI is necessary to be performed. The effect of these influence factors on the MSI design is also discussed.

First, the stiffness of the MSI with different piston areas is calculated, utilizing the mechanical model of MSI established in [21]; the result is plotted in Figure 4. We can see that the stiffness of the whole range increases with the increase in the piston area. In addition, the carrying capacity of the MSI also increases with the increase of the piston area. The phenomenon can be explained by the following reasons. The piston area has no influence on the volume change of the molecular spring material during the compression process. As a result, when the piston area increases, the displacement of the MSI decreases. Another reason is that, under the same environment condition, the working pressure of the molecular spring material is constant. Hence, the pressing force is proportional to the piston area. Consequently, the stiffness and the carrying capacity of the MSI increase with increasing piston area.

Macroscopically, the hydrophobicity of a porous material is typically characterized using the static water adsorption capacity instead of the contact angle. A low water adsorption 


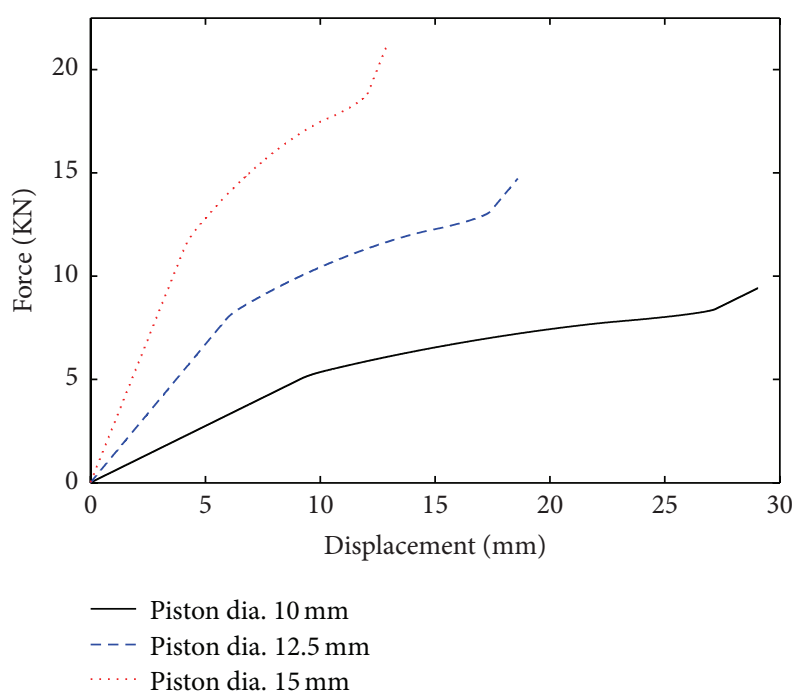

FIGURE 4: Theoretical force-displacement curves of the MSIs with different piston areas.

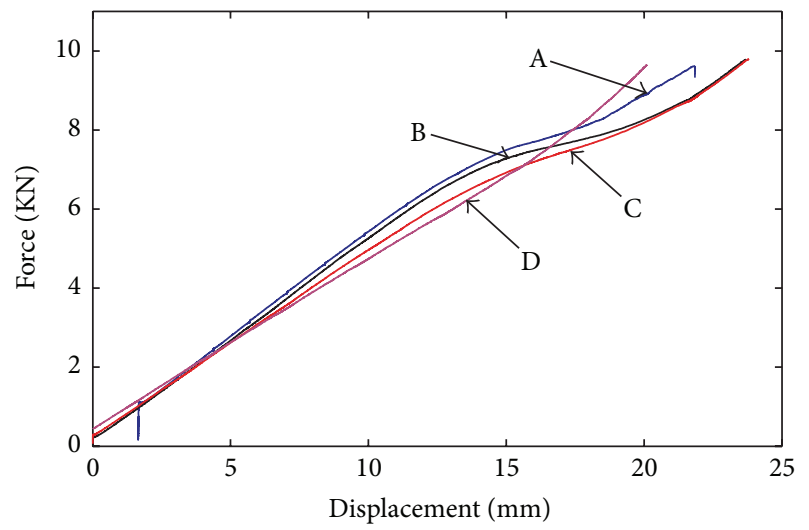

FIGURE 5: Experimental force-displacement curves of the MSI (ambient temperature: $10^{\circ} \mathrm{C}$ ). The Silicalite- 1 zeolites particles have different degrees of hydrophobicity (static water adsorption capacities are A, $0.68 \%$;, $1.08 \%$; C, $1.88 \%$; and D, $4.41 \%$, resp.).

capacity indicates strong hydrophobicity, that is, large contact angle. MSIs with Silicalite-1 zeolites of different water static adsorption capacity levels are tested in Figure 5. Figure 5 shows that the working pressure of the MSI decreases with increasing water adsorption capacity of the zeolites, as expected. However, the stiffness curve of material D is different from that of the others. The dynamic stiffness stage of material $\mathrm{D}$ is very narrow, and the piecewise property is not obvious because the hydrophobicity of material $\mathrm{D}$ is not as strong as that of the other three types of materials, with some of the pores even being hydrophilic. The higher hydrophilicity is considered to be caused by silanol defeats in the pores $[23,24]$. The hydrophilic pores never resist water and become saturated without compression; as a result, the effective porosity of zeolites decreases, leading to narrow dynamic stiffness stage of material D.

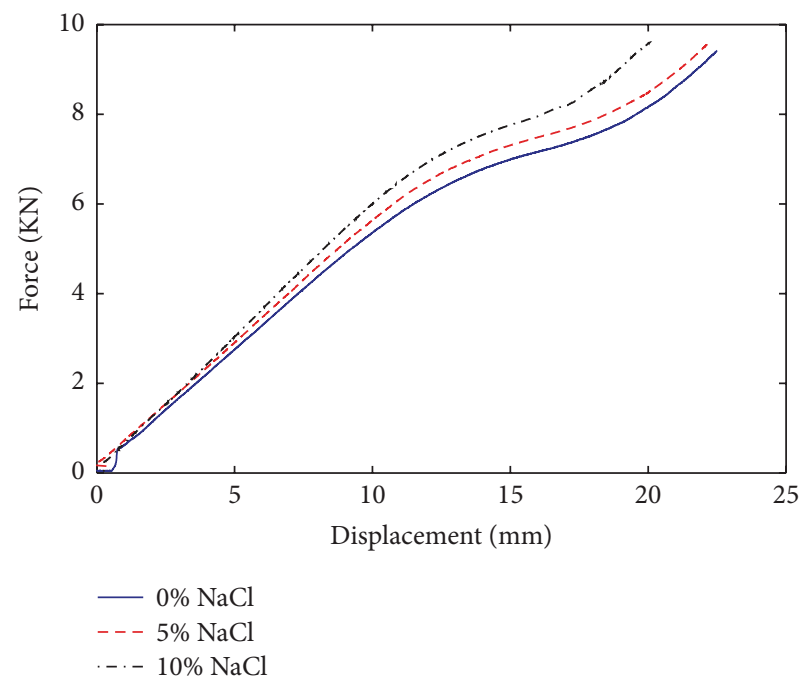

FIGURE 6: Experimental force-displacement curves with different concentrations of $\mathrm{NaCl}$ (7.5 g of Silicalite-1 filled; ambient temperature: $25^{\circ} \mathrm{C}$ ).

Note that ions also have an influence on the molecular spring. Three systems with different $\mathrm{NaCl}$ concentrations were studied in Figure 6. We can observe that the stiffness across the whole range increases with the concentration of $\mathrm{NaCl}$ rising. That is because the ions lead to stronger intermolecular interaction and make it more difficult to compress water. Meanwhile, the working pressure of the MSI also rises and this phenomenon might be attributed to the fact that $\mathrm{NaCl}$ solution exhibits higher surface tension than pure water [9]. Coincidentally, in [25], Michelin-Jamois et al. also find that electrolyte solutions can exhibit higher working pressure due to osmotic effect. And they quantitatively analyze the influence of $\mathrm{NaCl}$ and other electrolyte solutions on MSI. Thanks to that, the $\mathrm{NaCl}$ admixture can serve as an antifreezing agent, and the system can be used at temperatures lower than $0^{\circ} \mathrm{C}$.

On the contrary, some surface active agents help to decrease the surface tension of water. Figure 7 plots the force-displacement curve of a MSI with $1 \%$ octyl phenoxy polyethoxy solution as the working medium. We find that the working pressure of a MSI with a surface active agent decreases.

Endurance tests are performed to investigate the stability of the zeolites. A 100000-cycle sinusoidal test is applied to the MSI. The sinusoid excitation acting on the isolator is $10 \mathrm{~Hz}$, with an amplitude of $2 \mathrm{~mm}$, and the median of the excitation force is $8 \mathrm{kN}$. Figure 8 illustrates the force-displacement curves before and after the 100000-timesinusoidal tests. A good stable property of the zeolite material is confirmed by the result. We collected and dried the Silicalite-1 zeolite particles from the cylinder after three cycles of loadingunloading tests and endurance tests, respectively, as shown in Figure 9. Before each test, all of the particles are spherical. However, only after three cycles of the tests, some particles cracked into pieces (Figure 9(a)). Finally, after the 100000cycle endurance tests, most particles were crushed into 


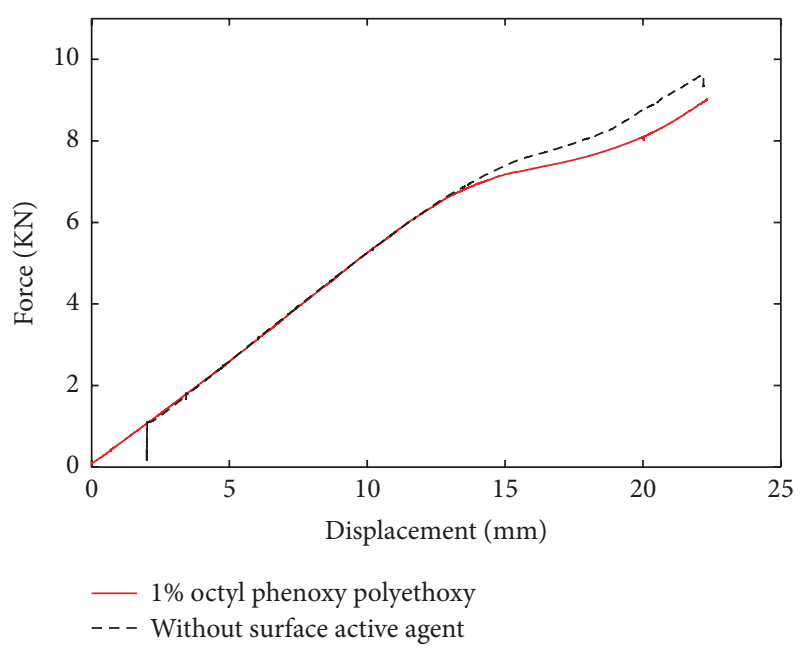

FIGURE 7: Experimental force-displacement curves of the MSI with and without a surface active agent (7.5 g of Silicalite-1 filled; ambient temperature: $5^{\circ} \mathrm{C}$ ).

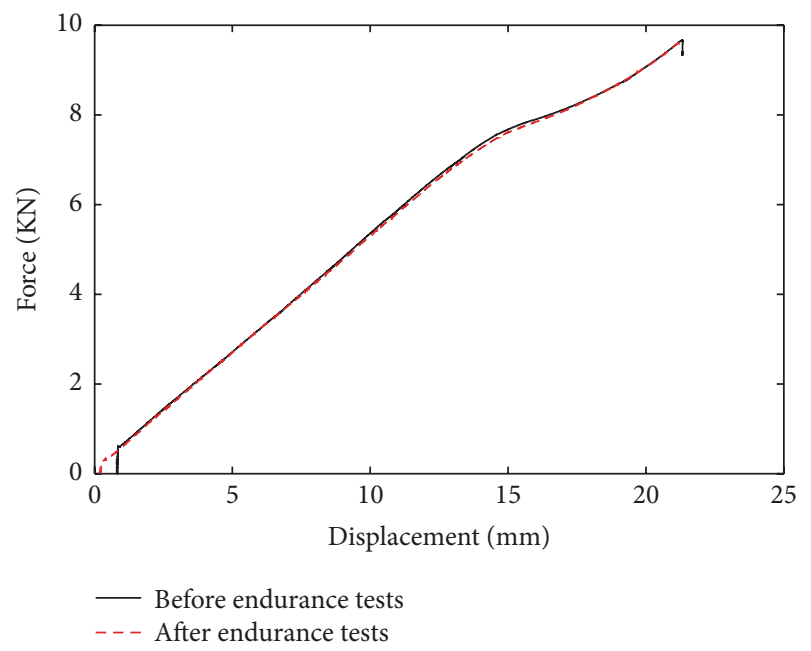

FIGURE 8: Experimental force-displacement curves before and after the endurance tests ( $5 \mathrm{~g}$ of Silicalite- 1 filled; ambient temperature: $\left.5^{\circ} \mathrm{C}\right)$.

fine particles (Figure 9(b)). However, due to the nanosized structures of the hydrophobic pores, the cracked particles do not change their nanostructures so that the stiffness properties of the MSI remain unchanged.

From the aspect of application, the properties of MSI can be conveniently adjusted for different engineering requirements. For example, during the design of MSI, the carrying capacity or stiffness can be controlled by changing the piston area. Once the MSI has been manufactured, the structure parameters cannot be changed anymore. The carrying capacity of the MSI can also be easily altered by adding ions or surface active agents or by changing the zeolite material. In addition, the stiffness of the MSI can be adjusted by increasing or decreasing the quantity of zeolites.

\section{FRFs of the Primary Resonance}

A single degree of freedom system supported by a MSI is given in Figure 10. On the static equilibrium condition, with the static load of the mass lump, the MSI is positioned within the range of dynamic stiffness (see Figure 11). Thus, the MSI vibration isolation system has High-Static-Low-Dynamic stiffness properties. Taking the static equilibrium position as the origin of the coordinate system, the equation of motion (EOM) of a MSI system subject to sinusoid excitation is

$$
M \ddot{x}+c \dot{x}+f_{k}(x)=F \cos (\omega t) .
$$

The static stiffness and stopping stiffness of MSI are linear because the compression ratio of water is linear. In contrast, the MSI has nonlinear dynamic stiffness intrinsically because the process of water intrusion-extrusion hydrophobic nanosized pores is very complicated, which produces a nonlinear behaviour. Through curve fitting, the elastic restoring force can be written as [21]

$$
f_{k}(x)= \begin{cases}k_{0} x+F c_{1} & \left(x>x_{1}\right) \\ \alpha_{1} x+\alpha_{2} x^{2}+\alpha_{3} x^{3} & \left(x_{2}<x<x_{1}\right) \\ k_{0} x+F c_{2} & \left(x<x_{2}\right),\end{cases}
$$

where $x_{1}$ and $x_{2}$ are critical displacements; $\alpha_{1}, \alpha_{2}$, and $\alpha_{3}$ are dynamic stiffness coefficients; and $k_{0}$ is the static stiffness and stopping stiffness. $F c_{1}$ and $F c_{2}$ are constant:

$$
\begin{aligned}
& F c_{1}=\left(\alpha_{1}-k_{0}+\alpha_{2} x_{1}+\alpha_{3} x_{1}^{2}\right) \cdot x_{1}, \\
& F c_{2}=\left(\alpha_{1}-k_{0}+\alpha_{2} x_{2}+\alpha_{3} x_{2}^{2}\right) \cdot x_{2} .
\end{aligned}
$$

To evaluate the FRFs of primary resonance of MSI vibration isolation system, a matching method based on EPL system [26] is utilized. We first transform the complicated piecewise-nonlinear system into an EPL system. Thereafter, we apply an exact method, that is, the matching method, to the EPL system. The conditions of periodicity, symmetry (if any), and consistency are maintained at every junction of adjacent linear ranges to solve the FRFs.

The EOM of EPL system has the same form as (2), except that $f_{k}(x)$ is replaced by a piecewise linear $f_{k_{e}}(x)$ as

$$
f_{k_{e}}(x)= \begin{cases}k_{0} x+F c_{1 e} & \left(x>x_{1}\right) \\ k_{e} x & \left(x_{2}<x<x_{1}\right) \\ k_{0} x+F c_{2 e} & \left(x<x_{2}\right)\end{cases}
$$

where $k_{e}$ is an unknown constant and

$$
\begin{aligned}
& F c_{1 e}=\left(k_{e}-k_{0}\right) \cdot x_{1}, \\
& F c_{2 e}=\left(k_{e}-k_{0}\right) \cdot x_{2} .
\end{aligned}
$$

Figure 12 gives a typical periodic solution of the EPL system having a single crossing per cycle, of which $t_{p}$ is equal to forcing period (i.e., $t_{p}=2 \pi / \omega$ ). We arbitrarily set $t=0$ when the system moves to $o$ point (see Figure 12), that is, 


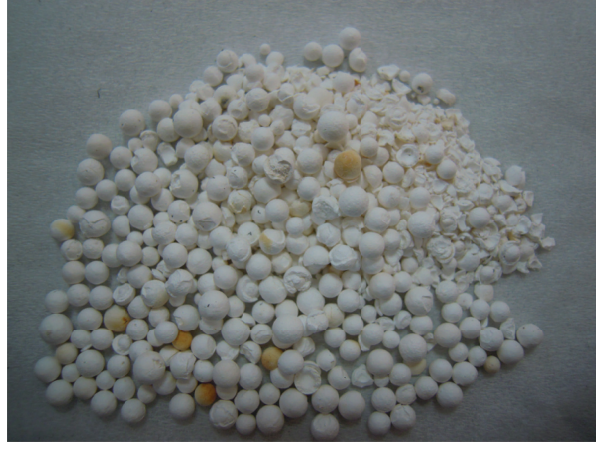

(a) After three cycles of the loading-unloading tests

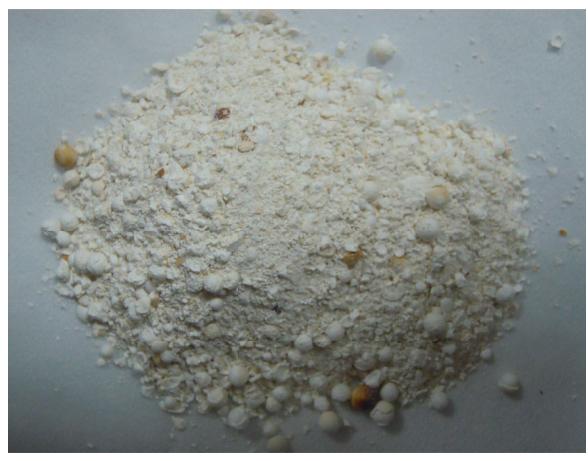

(b) After 100000-cycle endurance tests

FIGURE 9: Silicalite-1 zeolites particles after the experiment.

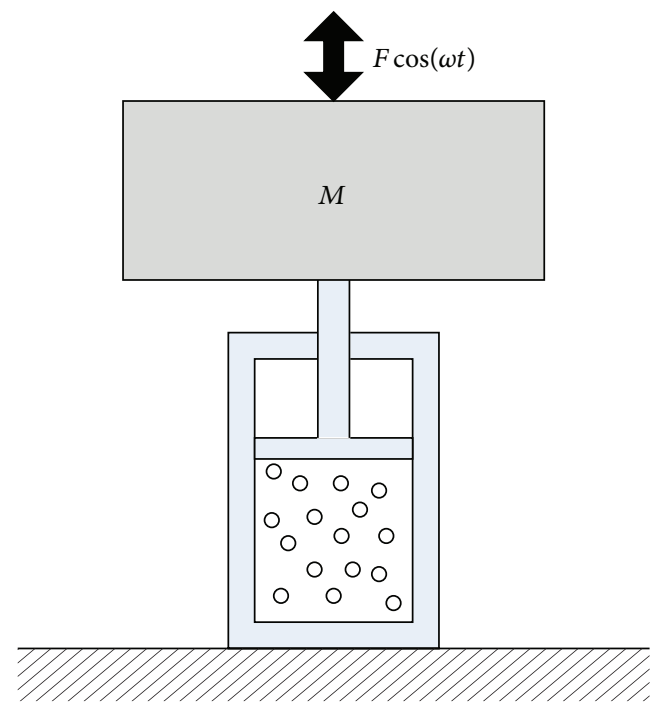

FIGURE 10: MSI vibration isolation system.

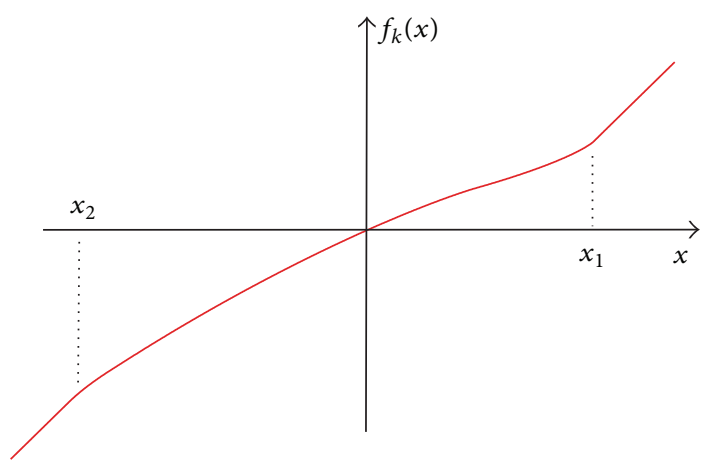

FIGURE 11: Stiffness curve of the MSI vibration isolation system; $x_{1}$ and $x_{2}$ are critical values of displacement, $x_{1}<-x_{2}$.

$x_{1}$ position with positive velocity. This setting introduces an unknown phase $\varphi$ in the excitation force; thus, the EOM of EPL system is rewritten as

$$
M \ddot{x}+c \dot{x}+f_{k_{e}}(x)=F \cos (\omega t+\varphi) .
$$

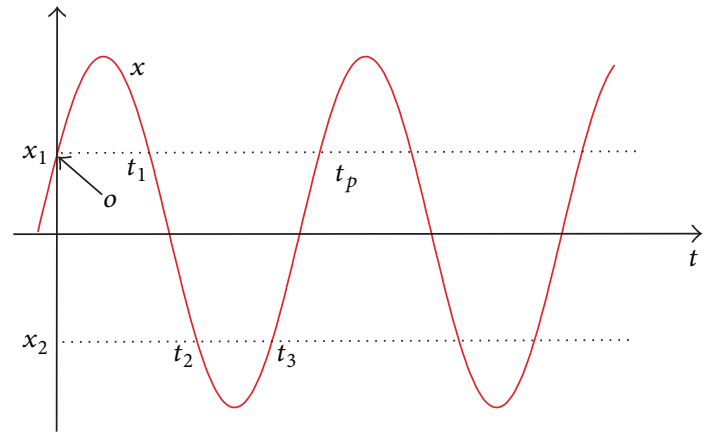

FIgURE 12: Periodic response in the time domain having a single crossing per cycle.

Therefore, a solution in the form shown in Figure 12 is written as

$$
\begin{array}{r}
X(t)=X_{1}(t) \cdot U\left(-T_{1}\right)+X_{2}(t) \cdot U\left(T_{1}\right) \cdot U\left(-T_{2}\right) \\
+X_{3}(t) \cdot U\left(T_{2}\right) \cdot U\left(-T_{3}\right)+X_{4}(t) \cdot U\left(T_{3}\right) \\
\left(0<t \leq t_{p}\right),
\end{array}
$$

where $T_{1}=t-t_{1}, T_{2}=t-t_{2}, T_{3}=t-t_{3}$, and

$$
U(T)= \begin{cases}0, & T<0 \\ 1, & T \geq 0 .\end{cases}
$$

$X_{1}, X_{2}, X_{3}$, and $X_{4}$ can be solved from the following equations:

$$
\begin{gathered}
M \ddot{X}_{1}+c \dot{X}_{1}+k_{0} X_{1}=F \cos (\omega t+\psi)-F c_{1}, \\
0<t \leq t_{1}, \\
M \ddot{X}_{2}+c \dot{X}_{2}+k_{e} X_{2}=F \cos (\omega t+\psi), \quad t_{1}<t \leq t_{2}, \\
M \ddot{X}_{3}+c \dot{X}_{3}+k_{0} X_{3}=F \cos (\omega t+\psi)-F c_{2}, \\
t_{2}<t \leq t_{3}, \\
M \ddot{X}_{4}+c \dot{X}_{4}+k_{e} X_{4}=F \cos (\omega t+\psi), \quad t_{3}<t \leq t_{p} .
\end{gathered}
$$


The solutions of (10) are

$$
\begin{aligned}
X_{1} & \\
= & e^{-\rho t}\left(A_{1} \cos \left(\omega_{1} t\right)+B_{1} \sin \left(\omega_{1} t\right)\right) \\
& +g_{1} \sin (\omega t+\psi)+h_{1} \cos (\omega t+\psi)-\frac{F c_{1}}{k_{0}},
\end{aligned}
$$

in which

$$
\begin{aligned}
\rho & =\frac{c}{2 M}, \\
\omega_{1} & =\sqrt{\frac{k_{0}}{M}} \cdot \sqrt{1-\frac{\rho M}{k_{0}}}, \\
\omega_{2} & =\sqrt{\frac{k_{e}}{M}} \cdot \sqrt{1-\frac{\rho M}{k_{e}}}, \\
g_{i} & =\frac{F \omega \rho}{M\left(\omega^{4}+\left(4 \rho^{2}-2 \omega_{i}^{2}\right) \omega^{2}+\omega_{i}^{4}\right)}, \\
h_{i} & =\frac{F\left(\omega_{i}^{2}-\omega^{2}\right)}{M\left(\omega^{4}+\left(4 \rho^{2}-2 \omega_{i}^{2}\right) \omega^{2}+\omega_{i}^{4}\right)},
\end{aligned}
$$

Except for $k_{e}$, there are 12 unknowns in (11), including $A_{i}$, $B_{i}(i=1,2,3,4), t_{1}, t_{2}, t_{3}$, and $\varphi$. Based on the matching method, the initial condition and continuity condition also supply 12 independent equations:

$$
\begin{aligned}
& X_{1}(0)=X_{1}\left(t_{1}\right)=X_{2}\left(t_{1}\right)=X_{4}\left(t_{p}\right)=x_{1}, \\
& X_{2}\left(t_{2}\right)=X_{3}\left(t_{2}\right)=X_{3}\left(t_{3}\right)=X_{4}\left(t_{3}\right)=x_{2}, \\
& \dot{X}_{1}\left(t_{1}\right)=\dot{X}_{2}\left(t_{1}\right), \\
& \dot{X}_{2}\left(t_{2}\right)=\dot{X}_{3}\left(t_{2}\right), \\
& \dot{X}_{3}\left(t_{3}\right)=\dot{X}_{4}\left(t_{3}\right), \\
& \dot{X}_{4}\left(t_{p}\right)=\dot{X}_{1}(0) .
\end{aligned}
$$

Thus, the complete response history of the system can be obtained in closed form if we can compute the value of $k_{e}$.

An approximation solution of $k_{e}$ can be solved by minimizing the integral

$$
L=\int_{0}^{t_{p}}\left(f_{k}(x)-f_{k_{e}}(x)\right)^{2} d t
$$

that is,

$$
\frac{\partial L}{\partial k_{e}}=\frac{\partial}{\partial k_{e}} \int_{0}^{t_{p}}\left(f_{k}(x)-f_{k_{e}}(x)\right)^{2} d t=0 .
$$

If we ignore the small difference between the values of $F c_{j}$ and $F c_{j e}(j=1,2),(15)$ is rewritten as

$$
\begin{aligned}
& \frac{\partial}{\partial k_{e}}\left(\int_{t_{1}}^{t_{2}}\left(\alpha_{1} X^{*}+\alpha_{2} X^{* 2}+\alpha_{3} X^{* 3}-k_{e} X^{*}\right)^{2} d t\right. \\
& \left.\quad+\int_{t_{3}}^{t_{p}}\left(\alpha_{1} X^{*}+\alpha_{2} X^{* 2}+\alpha_{3} X^{* 3}-k_{e} X^{*}\right)^{2} d t\right)=0,
\end{aligned}
$$

where $X^{*}(t)$ is the Fourier series of $X$ in (16):

$$
X^{*}(t)=d_{0}+d_{1} \cos \left(\omega t+\eta_{1}\right)+\cdots
$$

Substituting the first-order approximation $X^{*}(t)$ into (16) and considering the 12 independent equations of initial condition and continuity condition, the value of $k_{e}$ can be solved. Once $k_{e}$ is solved, the response history of the system can also be obtained from (11).

Figure 13 gives the frequency response of MSI system calculated by the matching method based on the EPL system and the numerical simulation (fourth-order Runge-Kutta method). The result shows that MSI exhibits hardening stiffness characteristics and the matching method based on EPL system has good precision to evaluate the FRFs of MSI isolation system, especially for small amplitude vibration. In this section, equivalent damping is utilized here (we define $c=730 \mathrm{Ns} \mathrm{m}^{-1}$ ); an improved damping model could be exploited in future work.

\section{Vibration Isolation Performance}

To evaluate the vibration isolation performance, the force transmissibility of MSI system is studied. For a nonlinear system, a harmonic external excitation may generate subharmonics, superharmonics, and even chaos. Hence, the traditional force transmissibility is not valid for a nonlinear system. In this section, the ratio of the root mean square values of the transmitted force and the excitation force is considered as force transmissibility of the nonlinear system; that is,

$$
T_{f}=20 \log _{10}\left(\frac{\operatorname{RMS}\left[f_{t}(t)\right]}{\operatorname{RMS}\left[f_{0}(t)\right]}\right),
$$




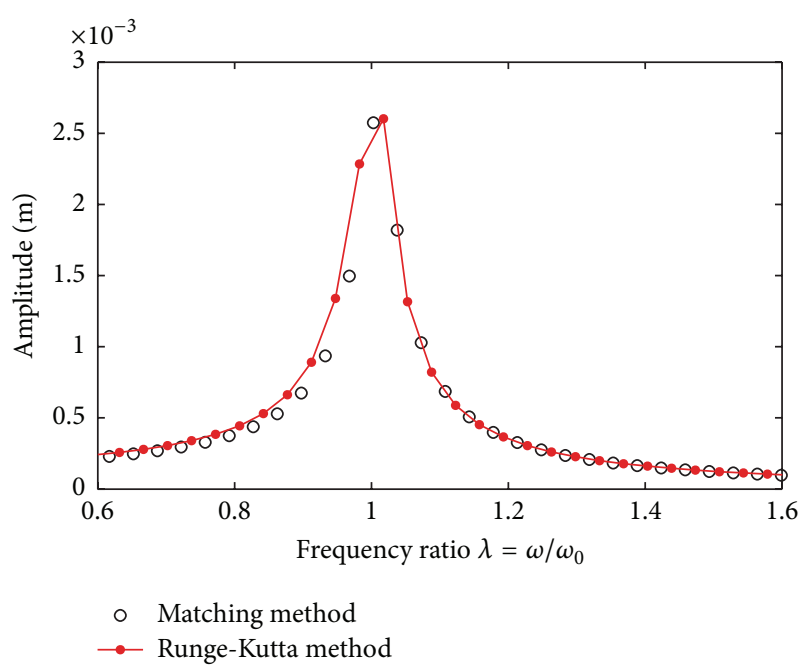

(a)

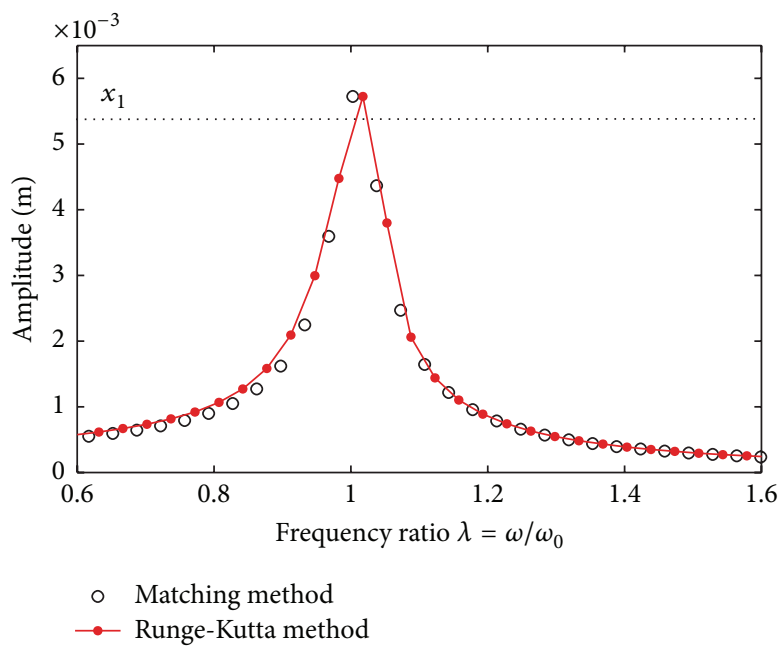

(b)

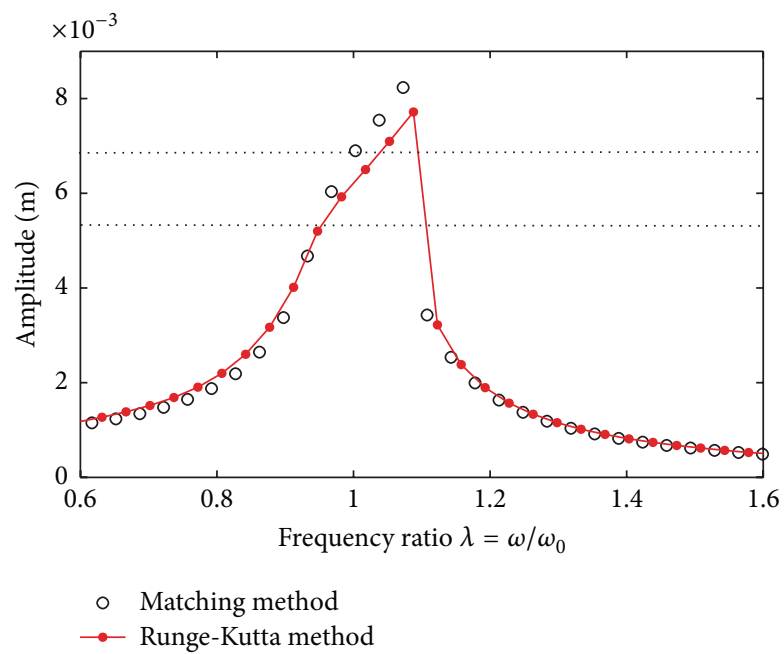

(c)

FIGURE 13: Frequency response of the MSI system; the system parameters are $k_{0}=5.494 \times 10^{5} \mathrm{Nm}^{-1}, \alpha_{1}=2.23411 \times 10^{5} \mathrm{Nm}^{-1}, \alpha_{2}=-8.5079 \times$ $10^{-6} \mathrm{Nm}^{-1}, \alpha_{3}=8.7465 \times 10^{8} \mathrm{Nm}^{-1}, c=730 \mathrm{Ns} \mathrm{m}^{-1}, x_{1}=5.3 \times 10^{-3} \mathrm{~m}, x_{2}=-6.7 \times 10^{-3} \mathrm{~m}, M=730 \mathrm{~kg}$, and $k_{e}=2.63 \times 10^{5} \mathrm{Nm}^{-1}$. Excitation force: (a) $F / M=0.05$; (b) $F / M=0.12$; (c) $F / M=0.25$.

where $f_{0}(t)$ is the excitation force and $f_{t}(t)$ is the transmitted force. The transmitted force can be replaced by the sum of restoring force and the damping force as well:

$$
\begin{aligned}
f_{t} & =f_{k}+c \dot{x} \\
& = \begin{cases}k_{0} x+F c_{1}+c \dot{x} & \left(x>x_{1}\right) \\
\alpha_{1} x+\alpha_{2} x^{2}+\alpha_{3} x^{3}+c \dot{x} & \left(x_{2}<x<x_{1}\right) \\
k_{0} x+F c_{2}+c \dot{x} & \left(x<x_{2}\right) .\end{cases}
\end{aligned}
$$

A numerical simulation (fourth-order Runge-Kutta method) is utilized to calculate the transmitted force of the MSI system. $f_{0}(t)$ and $f_{t}(t)$ from numerical simulation are discrete values; therefore, the root mean square can be derived as in the following equation:

$$
\begin{aligned}
& \operatorname{RMS}\left[f_{t}(t)\right]=\sqrt{\frac{\omega}{2 \pi} \sum_{j=0}^{N}\left[f_{t}\left(t_{j}\right)\right]^{2} \Delta t,} \\
& \operatorname{RMS}\left[f_{0}(t)\right]=\sqrt{\frac{\omega}{2 \pi} \sum_{j=0}^{N}\left[f_{0}\left(t_{j}\right)\right]^{2} \Delta t,}
\end{aligned}
$$

where $N$ is the sampling numbers.

By defining the force transmissibility in log-coordinates as

$$
L_{T}=20 \lg T_{f}
$$

a negative value of $L_{T}$ means the MSI isolates the vibration. Figure 14 shows a plot of $L_{T}$ of MSI with different quantities 
TABLE 1: Main parameters of the MSI with different quantities of zeolites.

\begin{tabular}{lcccc}
\hline Quantity of zeolites/g & $\begin{array}{c}k_{0} \\
\mathrm{Nm}^{-1}\end{array}$ & $\begin{array}{c}\alpha_{1} \\
\mathrm{Nm}^{-1}\end{array}$ & $\begin{array}{c}\alpha_{2} \\
\mathrm{Nm}^{-1}\end{array}$ & $\begin{array}{c}\alpha_{3} \\
\mathrm{Nm}^{-1}\end{array}$ \\
\hline 5 & $5.494 \times 10^{5}$ & $2.7141 \times 10^{5}$ & $-9.54347 \times 10^{-6}$ & $3.22077 \times 10^{8}$ \\
7.5 & $5.494 \times 10^{5}$ & $2.3411 \times 10^{5}$ & $-8.5079 \times 10^{-6}$ & $8.7465 \times 10^{8}$ \\
10 & $5.494 \times 10^{5}$ & $1.7909 \times 10^{5}$ & $-5.9740 \times 10^{-6}$ & $2.7149 \times 10^{8}$ \\
\hline
\end{tabular}

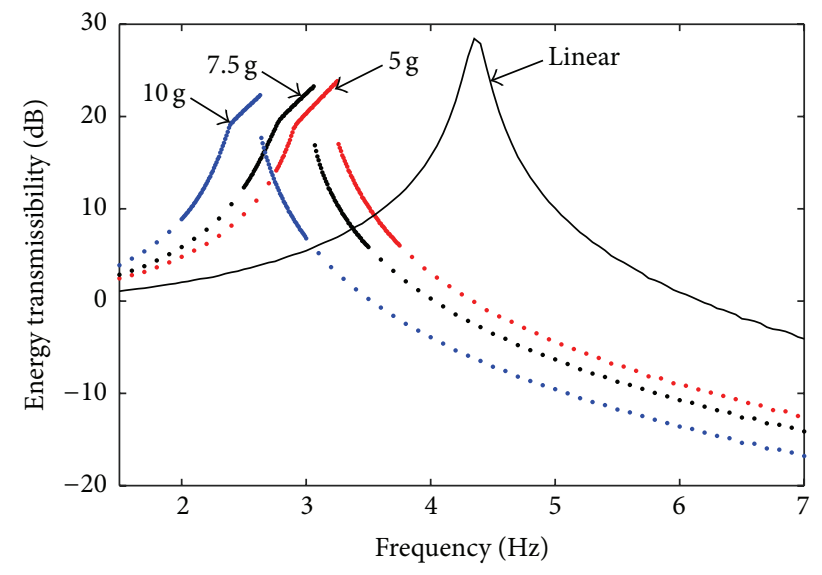

FIGURE 14: Force transmissibility curves of Molecular Spring Isolator system with different quantities of zeolites and a linear system $(F / M=0.18, M=730 \mathrm{~kg})$.

of zeolites compared with a linear system. The mentioned linear system possesses the same payload mass and damping coefficient with MSI isolation system and its stiffness is equal to the static stiffness of MSI. The main parameters of the MSI are given in Table 1.

Figure 14 shows that, compared with the linear system, the MSI systems exhibit a broader isolation bandwidth. The vibration isolation frequency starts at $3.5 \mathrm{~Hz}, 4 \mathrm{~Hz}, 4.3 \mathrm{~Hz}$, and $6.2 \mathrm{~Hz}$ for the MSI system with $10 \mathrm{~g}$ of zeolites, $7.5 \mathrm{~g}$ of zeolites, $5 \mathrm{~g}$ of zeolites, and a linear system, respectively. As expected, the MSI isolation system has better performance than linear system and isolation performance of MSI gets better with higher quantity of zeolites.

\section{Conclusions}

This paper investigated a novel vibration isolation technology using a molecular spring material, which consists of water and a hydrophobic nanoporous material. The MSI has a range of advantages over existing technologies: (i) tests and analysis prove that the MSI possesses sought-after HSLD stiffness for heavy equipment isolation application; (ii) the stiffness properties can be easily changed to maintain different engineering application, even after the MSI has been manufactured; and (iii) due to the special operational mechanism of the molecular spring material, the MSI performance is very stable, even if the particles are cracked.

For engineering applications, there is a primary obstacle to overcome: the design and choice of material of the container under high pressure up to $100 \mathrm{MPa}$. The pistoncylinder structure used in this paper is not a very durable design because of the difficulties in sealing at exceptionally high pressure. Moreover, the extremely high pressure also induces very high sealing damping. The best way to tackle these problems is to reduce the intrusion pressure of the hydrophobic nanoporous materials.

This paper mainly verifies the conceptual design of the MSI via a quasistatic test and an endurance test. The FRFs and force transmissibility of the MSI vibration systems were examined; the results suggest a very promising outcome. The above-mentioned obstacles make performing a dynamic experiment difficult; currently, an improved structural design of the MSI is under preparation for this experiment. The new design will eliminate the excessive damping caused by the seals. The results will be presented in the subsequent paper related to this work.

\section{Competing Interests}

The authors declare that they have no competing interests.

\section{Acknowledgments}

This work was supported by the National Natural Science Foundation of China under Grants nos. 11272145 and 11472127, the Funding of Jiangsu Innovation Program for Graduate Students under Grant no. CXLX13_134, the Fundamental Research Funds for the Central Universities, and a Project Funded by the Priority Academic Program Development of Jiangsu Higher Education Institutions (PAPD).

\section{References}

[1] X. Gao, Q. Chen, and H. D. Teng, "Modelling and dynamic properties of a novel solid and liquid mixture vibration isolator," Journal of Sound and Vibration, vol. 331, no. 16, pp. 3695-3709, 2012.

[2] X. Gao and Q. Chen, "Nonlinear frequency response analysis and dynamics design of a solid and liquid mixture nonlinear vibration isolator," Journal of Vibration and Control, vol. 20, no. 15, pp. 2389-2400, 2014.

[3] N. Zhou and K. Liu, "A tunable high-static-low-dynamic stiffness vibration isolator," Journal of Sound and Vibration, vol. 329, no. 9, pp. 1254-1273, 2010.

[4] A. Y. Fadeev and V. A. Eroshenko, "Study of penetration of water into hydrophobized porous silicas," Journal of Colloid and Interface Science, vol. 187, no. 2, pp. 275-282, 1997.

[5] C. V. Suciu, T. Iwatsubo, and S. Deki, "Investigation of a colloidal damper," Journal of Colloid and Interface Science, vol. 259, no. 1, pp. 62-80, 2003. 
[6] C. V. Suciu and K. Yaguchi, "Endurance tests on a colloidal damper destined to vehicle suspension," Experimental Mechanics, vol. 49, no. 3, pp. 383-393, 2009.

[7] T. Iwatsubo, K. Washio, H. Yano, and M. Miyazaki, "Experimental study of a colloidal damper to practical application," Journal of System Design and Dynamics, vol. 2, no. 5, pp. 1160-1169, 2008.

[8] T. W. Ku, S. B. Jeon, V. H. Bui, W. J. Song, M. S. Park, and B. S. Kang, "Numerical and experimental approach on energy dissipation in nano colloidal damper," Journal of Mechanical Science and Technology, vol. 21, no. 10, pp. 1464-1470, 2007.

[9] X. Kong, F. B. Surani, and Y. Qiao, "Energy absorption of nanoporous silica particles in aqueous solutions of sodium chloride," Physica Scripta, vol. 74, no. 5, pp. 531-534, 2006.

[10] F. B. Surani, X. Kong, D. B. Panchal, and Y. Qiao, "Energy absorption of a nanoporous system subjected to dynamic loadings," Applied Physics Letters, vol. 87, no. 16, Article ID 163111, pp. 1-3, 2005.

[11] A. Han, X. Kong, and Y. Qiao, "Pressure induced liquid infiltration in nanopores," Journal of Applied Physics, vol. 100, no. 1, Article ID 014308, pp. 1-3, 2006.

[12] V. Eroshenko, R.-C. Regis, M. Soulard, and J. Patarin, "Energetics: a new field of applications for hydrophobic zeolites," Journal of the American Chemical Society, vol. 123, no. 33, pp. 8129-8130, 2001.

[13] I. Khay, T. J. Daou, H. Nouali, A. Ryzhikov, S. Rigolet, and J. Patarin, "High pressure intrusion-extrusion of $\mathrm{LiCl}$ aqueous solutions in silicalite-1 zeolite: influence on energetic performances," The Journal of Physical Chemistry C, vol. 118, no. 8, pp. 3935-3941, 2014.

[14] M. Trzpit, S. Rigolet, and J. Paillaud, "Pure silica chabazite molecular spring: a structural study on water intrusionextrusion processes," The Journal of Physical Chemistry, vol. 118, pp. 3935-3941, 2014.

[15] L. Tzanis, M. Trzpit, M. Soulard, and J. Patarin, "High pressure water intrusion investigation of pure silica $1 \mathrm{D}$ channel AFI, MTW and TON-type zeolites," Microporous and Mesoporous Materials, vol. 146, no. 1-3, pp. 119-126, 2011.

[16] L. Tzanis, M. Trzpit, M. Soulard, and J. Patarin, "Energetic performances of channel and cage-type zeosils," Journal of Physical Chemistry C, vol. 116, no. 38, pp. 20389-20395, 2012.

[17] N. Desbiens, A. Boutin, and I. Demachy, "Water condensation in hydrophobic silicalite-1 zeolite: a molecular simulation study," Journal of Physical Chemistry B, vol. 109, no. 50, pp. 24071-24076, 2005.

[18] B. Lefevre, A. Saugey, J. L. Barrat et al., "Intrusion and extrusion of water in hydrophobic mesopores," Journal of Chemical Physics, vol. 120, no. 10, pp. 4927-4938, 2004.

[19] X. Chen, G. Cao, A. Han et al., "Nanoscale fluid transport: size and rate effects," Nano Letters, vol. 8, no. 9, pp. 2988-2992, 2008.

[20] A. Carrella, M. J. Brennan, T. P. Waters, and K. Shin, “On the design of a high-static-low-dynamic stiffness isolator using linear mechanical springs and magnets," Journal of Sound and Vibration, vol. 315, no. 3, pp. 712-720, 2008.

[21] M. C. Yu, Q. Chen, and X. Gao, “Theoretical and experimental investigation of molecular spring isolator," in Microsystem Technologies, 2015.

[22] L. B. McCusker, F. Liebau, and G. Engelhardt, "Nomenclature of structural and compositional characteristics of ordered microporous and mesoporous materials with inorganic hosts (IUPAC recommendations 2001)," Microporous and Mesoporous Materials, vol. 58, no. 1, pp. 3-13, 2003.
[23] M. Trzpit, M. Soulard, J. Patarin et al., "The effect of local defects on water adsorption in silicalite-1 zeolite: a joint experimental and molecular simulation study," Langmuir, vol. 23, no. 20, pp. 10131-10139, 2007.

[24] M. Trzpit, M. Soulard, J. Patarin et al., "Influence of defects on the water intrusion in silicalite-1 zeolite: confrontation of experimental and molecular simulation results," Studies in Surface Science and Catalysis, vol. 174, pp. 561-564, 2008.

[25] M. Michelin-Jamois, C. Picard, G. Vigier, and E. Charlaix, "Giant osmotic pressure in the forced wetting of hydrophobic nanopores," Physical Review Letters, vol. 115, no. 3, Article ID 036101, 2015.

[26] S. Chatterjee, A. K. Mallik, and A. Ghosh, "Periodic response of piecewise non-linear oscillators under harmonic excitation," Journal of Sound and Vibration, vol. 191, no. 1, pp. 129-144, 1996. 


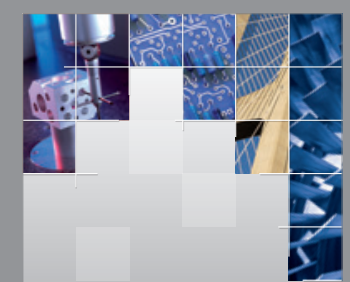

\section{Enfincering}
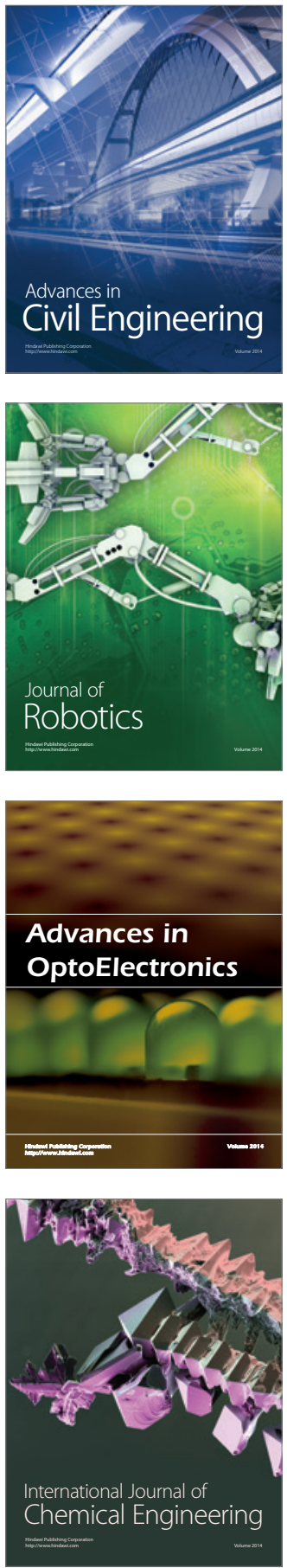

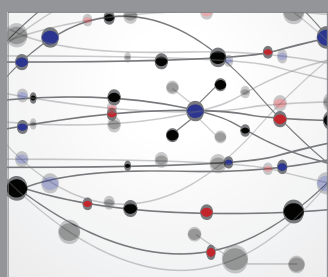

The Scientific World Journal

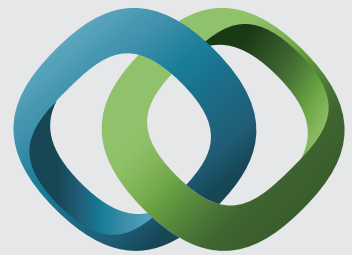

\section{Hindawi}

Submit your manuscripts at

http://www.hindawi.com
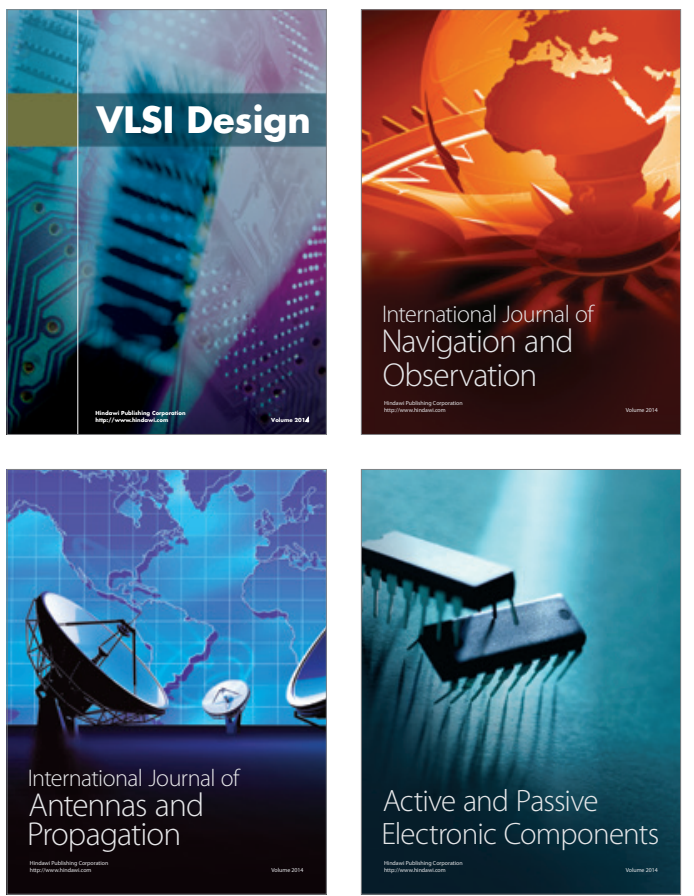
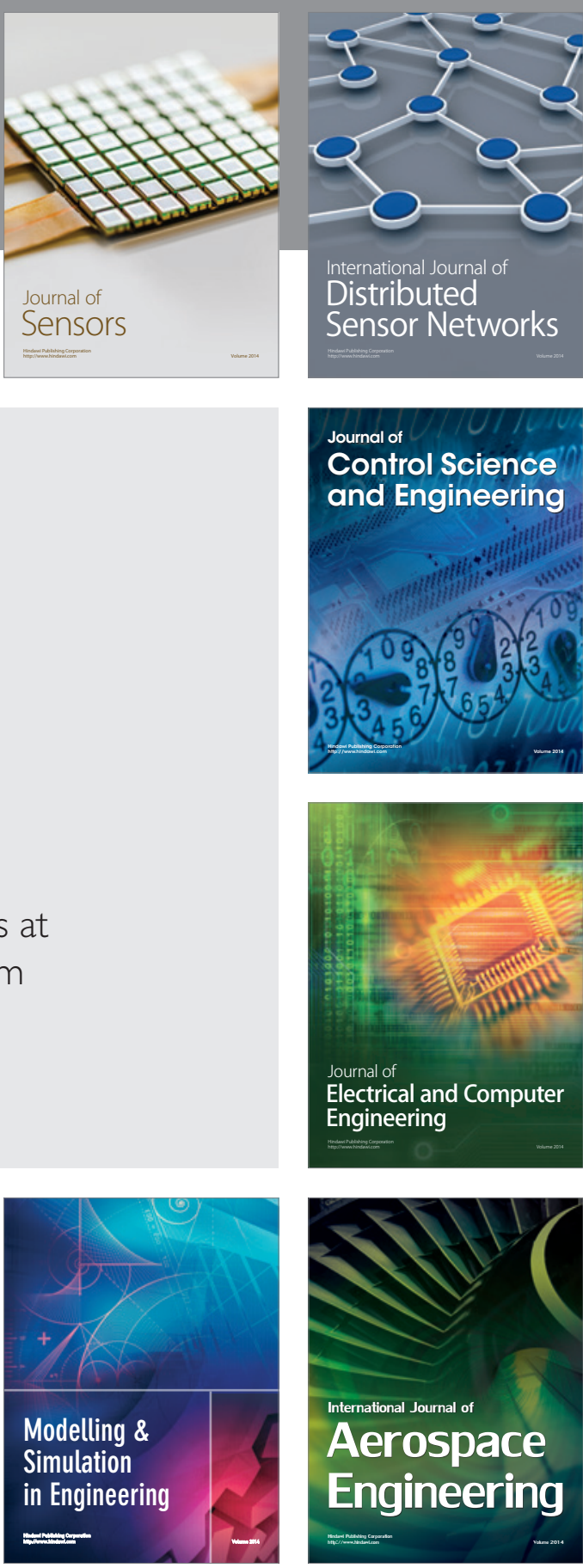

International Journal of

Distributed

Sensor Networks

Journal of

Control Science

and Engineering
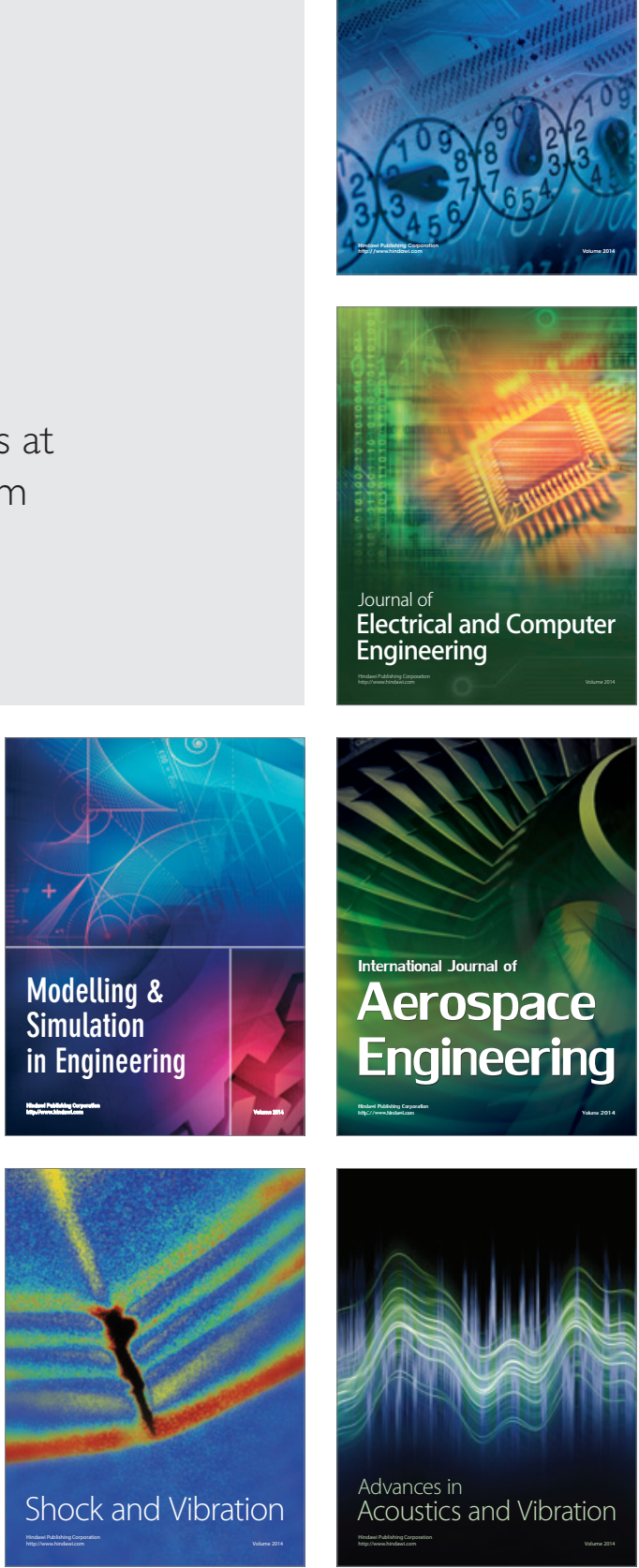